\title{
Explicit modeling of solid ocean floor in shallow underwater explosions
}

\author{
A.P. Walters, J.M. Didoszak* and Y.W. Kwon \\ Mechanical and Aerospace Engineering Department, Naval Postgraduate School, Monterey, CA, USA
}

Received 1 March 2012

Revised 6 July 2012

\begin{abstract}
Current practices for modeling the ocean floor in underwater explosion simulations call for application of an inviscid fluid with soil properties. A method for modeling the ocean floor as a Lagrangian solid, vice an Eulerian fluid, was developed in order to determine its effects on underwater explosions in shallow water using the DYSMAS solver. The Lagrangian solid bottom model utilized transmitting boundary segments, exterior nodal forces acting as constraints, and the application of prestress to minimize any distortions into the fluid domain. For simplicity, elastic materials were used in this current effort, though multiple constitutive soil models can be applied to improve the overall accuracy of the model. Even though this method is unable to account for soil cratering effects, it does however provide the distinct advantage of modeling contoured ocean floors such as dredged channels and sloped bottoms absent in Eulerian formulations. The study conducted here showed significant differences among the initial bottom reflections for the different solid bottom contours that were modeled. The most important bottom contour effect was the distortion to the gas bubble and its associated first pulse timing. In addition to its utility in bottom modeling, implementation of the non-reflecting boundary along with realistic material models can be used to drastically reduce the size of current fluid domains.
\end{abstract}

Keywords: Underwater explosion, ocean floor modeling, shallow water, fluid structure interaction

\section{Introduction}

The recent focus on naval operations in littoral waters, coupled with the delivery of the Littoral Combat Ship (LCS), presents unique challenges to the field of shock testing and simulation [1,2]. As full scale Underwater Explosion (UNDEX) testing of naval vessels has typically been conducted in deep water, the shallow water environment effects on the UNDEX response of a ship have not been extensively investigated [3]. The 2006 shock testing of the Ex-Lütjens in the Baltic Sea is one of the few documented tests performed in littoral waters in which the U.S. Navy conducted pre- and post- test computer simulations [4,5]. The simulation of this event was conducted with the Dynamic System Mechanics Advanced Simulation (DYSMAS) hydrocode [6]. DYSMAS was designed to model the fully-coupled, fluid-structure interaction problem of an UNDEX event on a ship [7]. It consists of three main programs: an Eulerian fluid solver, Gemini, a Lagrangian structural solver, Dyna_N(3D), and a Standard Coupler Interface between the two [8].

At its most fundamental level, the accepted bottom modeling method used in DYSMAS treats the soil as an inviscid fluid[9]. This research developed an alternative approach that utilizes the structural solver of DYSMAS to model the bottom as a solid, finite element structure. This Lagrangian solid bottom modeling approach was compared to the current bottom modeling technique to determine its validity and potential benefits. Whereas the current method can only create horizontal surfaces for all practical purposes, this Lagrangian solid bottom modeling method provides

\footnotetext{
*Corresponding author: J.M. Didoszak, Mechanical and Aerospace Engineering Department, Naval Postgraduate School, 700 Dyer Road, Monterey, CA 93943, USA. E-mail: jmdidosz@nps.edu.
} 


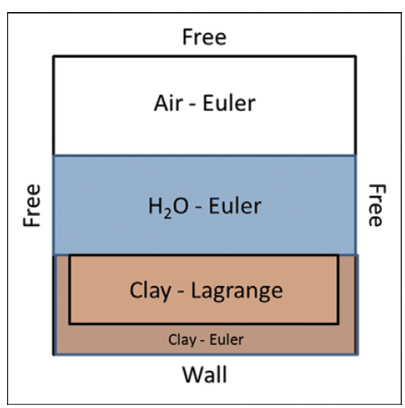

Fig. 1. Lagrangian solid bottom encased within Eulerian soil.

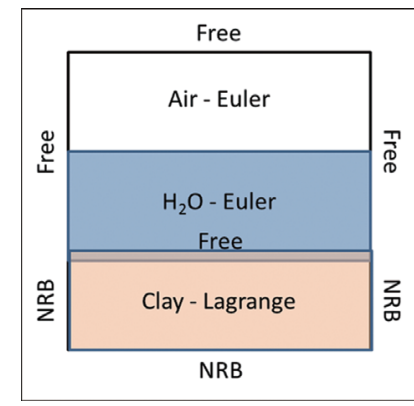

Fig. 2. Lagrangian solid bottom overlapped with fluid domain.

the capability to model contoured bottom profiles. A parametric study of this capability was conducted to determine the effect that contoured bottom profiles have on the response of a ship subjected to an UNDEX event in littoral waters.

\section{Solid ocean bottom modeling method}

There are two fundamentally different approaches to modeling the ocean bottom as a Lagrange solid interacting with an Eulerian fluid. The first method places the solid model entirely within the fluid domain as diagrammed in Fig. 1. The appeal of this method is in its ease of application. Since the entire structure is included within the fluid domain, the input deck requirements for the code are essentially the same as those for inserting the finite element model of a ship into the same domain. As shown in Fig. 1, the fluid domain boundaries were all modeled as "free" or non-reflecting boundaries with the exception of the absolute bottom of the fluid domain which was modeled as a "wall" or fully reflecting boundary. Implementation of the "free" boundary in the Gemini solver dictates that the same conditions exist on the other side of the border cells for running the calculation [10].

The major detractor of this method is the occurrence of retransmission from the solid bottom model. It was found that as the UNDEX shockwave was incident upon the bottom, a portion of the wave was reflected and the remainder transmitted into the model. The transmitted wave propagated through the model to the boundary displacement constraints, where it was reflected back through the model and into the fluid domain. This created multiple bottom reflections which were termed retransmission. Additionally, it proved difficult to precisely match the constitutive equations of the solid model to the surrounding Euler soil equation of state.

The drawbacks of the method described in Fig. 1 can to be resolved utilizing a solid model which was only partially included within the fluid domain as shown in Fig. 2. This method does not utilize an Eulerian soil model, rendering the need to match constitutive equations moot. An added benefit of eliminating the Euler clay is an associated decrease in fluid domain volume. The fluid domain is thus reduced by the volume of the cells beneath the Lagrange clay slab and to all four sides of the slab in a 3D model. This creates the potential to significantly decrease the required computational resources necessary in solving this type of problem.

By removing the need to interface all six sides of the solid bottom to the fluid domain, the model is able to incorporate transmitting, or non-reflecting, boundary segments (NRB) which effectively eliminated the retransmission effect. The NRB's were incorporated into the Dyna_N software to allow the simulation of semi-infinite structural domains. This feature is implemented with the application of normal and shear stresses on the exterior boundaries of the domain. The magnitude of these applied stresses is directly proportional to the velocity of the shockwave which is incident on the domain boundary [11]. This effectively cancels out the shockwave at the Lagrangian model boundaries and prevents false reflections from the bottom to the fluid. In addition, the NRB's enabled the vertical thickness of the model to be as few as two elements. Using such a small number of elements for the vertical thickness significantly reduces the computational cost in modeling the solid ocean bottom.

All simulations were executed on the Naval Postgraduate School's "Hamming" high performance computing cluster. This Linux based system is comprised of 1152 cores, running a minimum of AMD Opteron 2.2GHZ processors with 2 GB RAM per core. Table 1 provides a comparison of run times and other properties for three flat bottom 
Table 1

Comparison of flat bottom model computer simulation parameters

\begin{tabular}{|c|c|c|c|c|c|c|c|c|}
\hline Model & $\begin{array}{c}\text { Computational time } \\
\text { step (sec) }\end{array}$ & $\begin{array}{l}\text { Problem } \\
\text { time }(\mathrm{sec})\end{array}$ & $\begin{array}{l}\text { CPU time } \\
(\mathrm{sec})\end{array}$ & $\begin{array}{l}\text { Fluid cells } \\
\text { (million) }\end{array}$ & $\begin{array}{c}\text { Solid } \\
\text { elements }\end{array}$ & $\begin{array}{c}\text { Shell } \\
\text { elements }\end{array}$ & $\begin{array}{l}\text { \# CPU } \\
\text { (Fluid) }\end{array}$ & $\begin{array}{c}\text { \# CPU } \\
\text { (Structure) }\end{array}$ \\
\hline Euler Wall B.C. & $1.50 \mathrm{E}-05$ & 1 & $1.07 \mathrm{E}+05$ & 20.656 & 0 & 12792 & 48 & 1 \\
\hline Euler Clay & $2.24 \mathrm{E}-05$ & 1 & $1.49 \mathrm{E}+05$ & 21.061 & 0 & 12792 & 48 & 1 \\
\hline
\end{tabular}

cases. The first case has a wall boundary condition at the absolute bottom of the fluid domain. The second model contains an Euler clay bottom, while the third has a solid clay bottom using the Lagrangian modeling technique. The fluid volume consisted of approximately 21 million cells in each case. The shell elements represent the structure of the Floating Shock Platform (FSP) while the solid elements are the Lagrangian clay bottom. In each case there were approximately 20 million fluid cells and the problem was run out for a full second. Parallelization was consistent throughout with the use of 48 processors for the fluid domain and only a single CPU for all structural elements. The results show a $37 \%$ reduction in overall computational time, even with the addition of solid elements in the case of the Lagrangian solid bottom model.

For these reasons the second method was utilized for all of the follow-on simulations. However, resolution of two model-specific inaccuracies was still required. These were the vertical translation due to gravity and presence of an initial pressure wave resulting from settling out of the model at problem start. Typically either of these issues is easily handled by inclusion of appropriate boundary conditions and dynamic relaxation respectively. However, due to the uniqueness of the problem setup, these standard techniques were unable to be implemented.

The nature of the NRB segments prevented the application of displacement constraints on the solid model. The fixed value (Dirichelt) boundary conditions could not be applied simultaneously with the NRB segments which acted as flux (Neumann) boundary conditions. In order to prevent the vertical translation of the model due to gravity, an alternative solution was required. A solution was previously developed by O'Shea and Koltuniuk [12]. First a static solution for the solid model under hydrostatic pressure with nodal displacement constraints was determined. The reaction forces at displacement constraints were recorded and then applied to the dynamic model as constant external forces. The NRB segments were then applied to the dynamic model as well. This process allowed the hydrostatic pressure and self-weight loading of the model to be absorbed by the reaction forces and the transient shockwave loading was offset by the stress matching functions of the NRB segments. In this manner a complete force balance for the structural model was implemented. Utilizing this method, the maximum vertical translation that resulted in any simulation was approximately one centimeter. With a water column depth of 35 meters, this amount of translation was considered negligible and served to validate the method. All of the simulations in this research were done with a charge weight of 60 pounds $(27.2 \mathrm{~kg})$. Vertical translation should be reexamined in future simulations that include charges of significantly larger size. The second inaccuracy was the creation of a pressure wave emanating from the model into the fluid at the start of the simulation. This effect was determined to be the result of the model having been initially in an unstressed condition. The initial compression of the solid model created an associated low pressure wave which distorted the fluid domain response of the UNDEX. The dynamic relaxation tool of Dyna_N(3D), while effective for a stand-alone model, proved unable to relax the model once the ship structure model was included. Two alternatives were utilized to minimize this initial compression. The first was the application of the Prestress function which allowed the user to input an initial 3-D stress tensor for every element in the solid bottom. The stress tensors were calculated with the solution of the static structure. While best at minimizing the initial bottom wave, the Prestress function can only be applied to elastic or elastic-plastic material models. In the case of this research, the solid bottom was made with an elastic material model and the Prestress function was used. Since future research may employ complex material models, an additional method to eliminate the bottom wave was developed. The second method was to let the coupled simulation run with no explosive event for a minimum of 2.5 seconds. It was found that at this point the kinetic energy was reduced to less than $1.3 \mathrm{E}-08 \mathrm{~J} / \mathrm{g}$ while the strain in the vertical direction was found to be $1.2 \mathrm{E}-06$, while the change in velocity was approximately $0.001 \mathrm{~cm} / \mathrm{sec}$. This effectively put the solid model in a state near to static equilibrium prior to the start of the transient UNDEX event. 


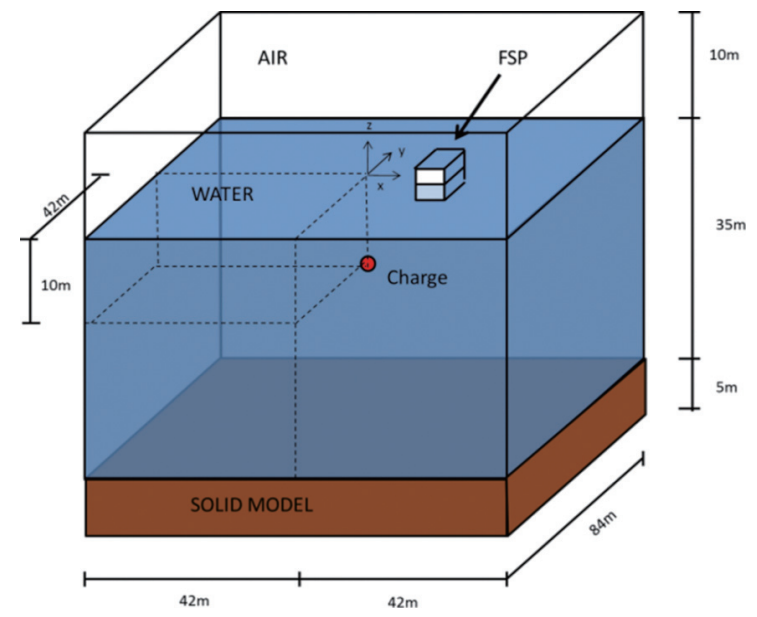

Fig. 3. Representative DYSMAS Simulation Geometry.

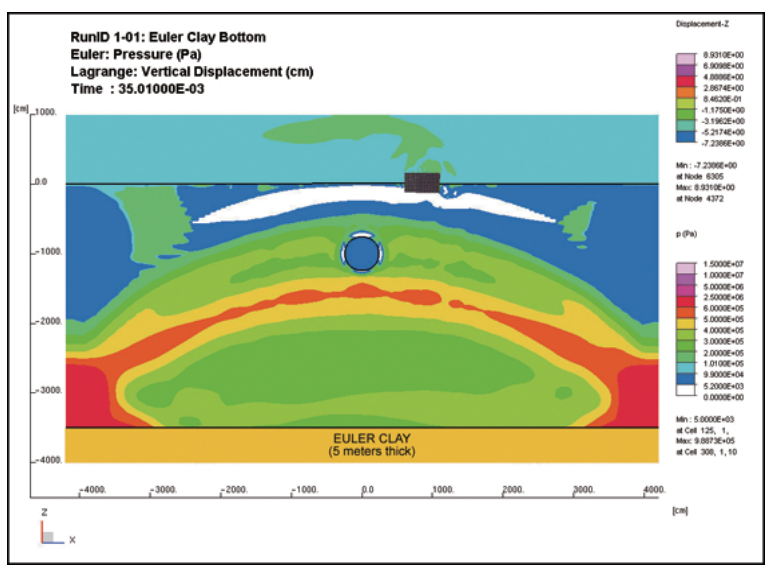

Fig. 5. Pressure Field at $35 \mathrm{msec}$ for an Euler Clay Bottom.

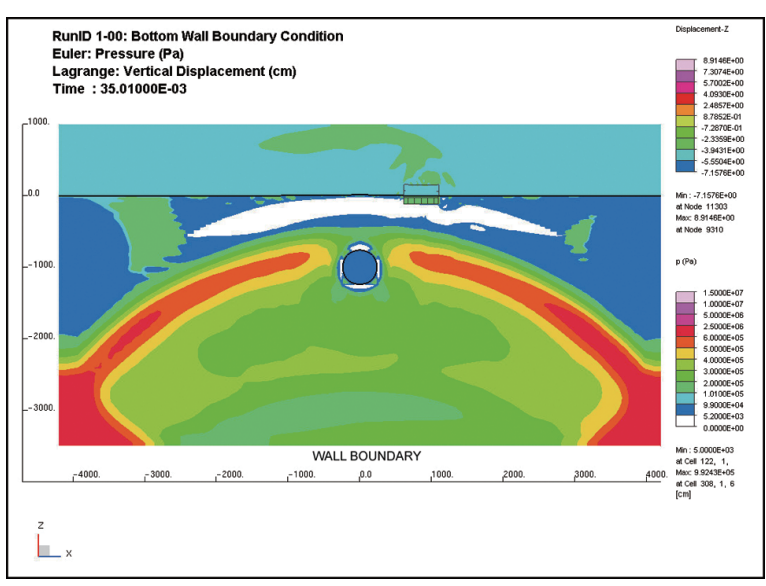

Fig. 4. Pressure Field at $35 \mathrm{msec}$ for an Euler Wall Boundary Condition.

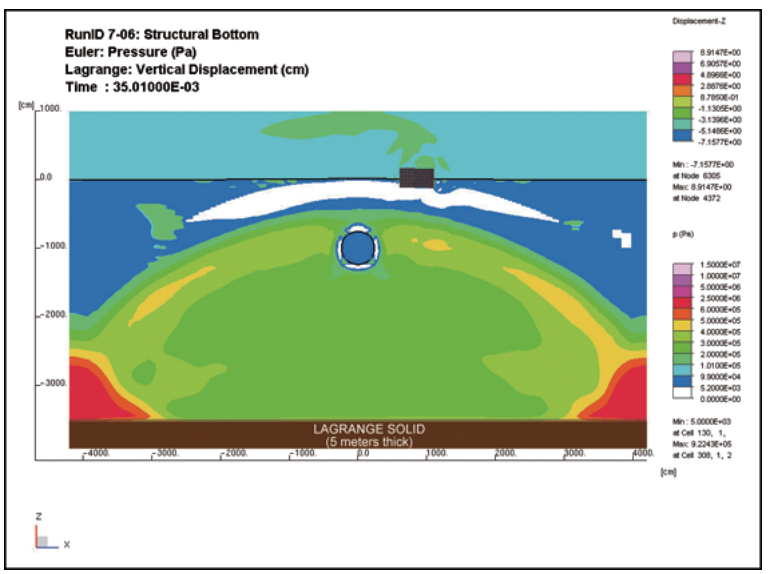

Fig. 6. Pressure Field at $35 \mathrm{msec}$ for a Lagrangian Solid Bottom.

\section{Comparison to current bottom modeling methods}

There are two current practices for modeling the ocean bottom in DYSMAS. The simplest method is the application of a perfectly reflective boundary condition on the bottom of the fluid domain, which creates an ocean floor that absorbs zero energy in an UNDEX event. This was called the Euler Wall model. A more accurate Eulerian technique was developed by creating a fluid layer with either a Mie-Grüneisen or P-alpha equation of state with the properties of various clay or sand ocean bottoms [9]. This model was called the Euler Clay model. In order to validate the solid bottom model technique, this research compared the simulated responses of the same event for each method. As shown in Fig. 3, the computational volume used was 84 meters square laterally and 35 meters deep. A five meter thick layer of air was placed above the water. The bottom thicknesses were set to five meters for both the Eulerian and the Lagrangian bottom models. The charge was placed in the lateral center of the water at a depth of ten meters. The desired outcome of the comparison was to qualitatively match the bottom reflection shockwaves and the gas bubble response of the three bottom modeling methods.

The bottom reflection shockwave responses were compared 35 milliseconds after the charge was detonated. This time was chosen in order to capture the entire bottom reflection response prior to the reflected shockwave impacting the bulk cavitation zone. Figures 4, 5 and 6 are the flow field pressure plots for the Euler wall boundary condition, the Euler clay, and the Lagrangian solid bottom respectively. Figure 7 provides the pressure profile through the depth of the water column for all three simulations. Of note is the clear correlation in shape between the Euler wall 
Table 2

Maximum radius and first bubble pulse

\begin{tabular}{lccc}
\hline Model & Bubble radius $(\mathrm{cm})$ & $1^{\text {st }}$ Pulse time $(\mathrm{msec})$ & $1^{\text {st }}$ Pulse pressure $(\mathrm{Pa})$ \\
\hline Euler Wall B.C. & $475 \mathrm{~cm}$ & 563 & $6 \times 10^{5}$ \\
Euler Clay & $482 \mathrm{~cm}$ & 576 & $12 \times 10^{5}$ \\
Lagrangian solid bottom & $475 \mathrm{~cm}$ & 563 & $7 \times 10^{5}$ \\
\hline
\end{tabular}

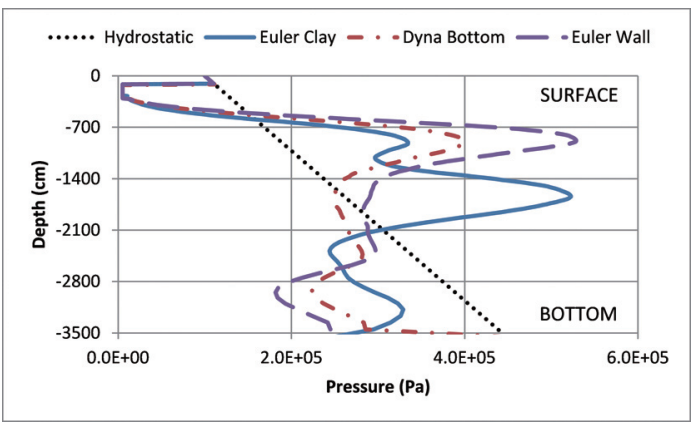

Fig. 7. Bottom reflection pressure comparison.

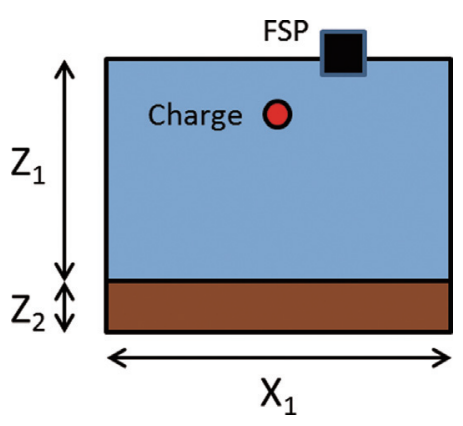

Fig. 8. Flat.

boundary condition (dashed line) and the Lagrangian solid bottom (dash-dot line). The only difference between the two appears to be the magnitude of the reflected pressures. This demonstrates that the Lagrangian solid bottom absorbed a portion of the energy from the UNDEX event. The Euler clay (solid line) follows the same pattern from the surface to 1000 centimeters deep. At this point the response diverges from the previous simulation with a large pressure spike at 1600 centimeters. This second, larger reflection is the result of the wall boundary condition which was required beneath the Eulerian clay layer. This result was previously noted by Didoszak, Prendergast and Shin [13]. The wave was minimized by increasing the thickness of the Eulerian clay layer. This allowed the shockwave energy to dissipate thoroughly before it returned to the water column. With the elimination of the second reflection, the expectation is that the pressure profile response of the Eulerian clay would then be comparable to the previous simulations. However, the drawback is a considerable increase in required computational resources required for this increased volume [14]. Therefore the bottom thickness was kept at five meters to easily compare it to the five meter thick Lagrangian solid bottom. Comparing the magnitudes of the initial reflections, the wall condition provides the greatest pressure magnitude reflection and the Euler clay provides the least. A better match between the Euler clay and Lagrangian solid bottom could be achieved by adjusting the material properties used in the Lagrangian solid bottom.

The bottom model type also affected the bubble response. Table 2 compares the response of each simulation's bubble and associated first pulse. Once again the Lagrangian solid bottom closely follows the wall boundary condition in all areas. The significant outlier is the incident pressure of the Euler clay's first pulse, which is nearly double the incident pressure of the other two models.

The qualitative differences between the Lagrangian solid bottom model and the Euler clay layer are minimal. The bottom reflection shockwave response differences can be mitigated through an increased thickness in the Euler clay layer and the application of more accurate material properties to the Lagrangian solid bottom. The differences in the gas bubble responses could not be accurately accounted for. Further simulations utilizing the previously mentioned adjustments may provide a more consistent comparison between the bubbles responses.

\section{Shallow water bottom contour effects}

Modeling the bottom as a structure in Dyna_N provides the capability to create bottoms which are not flat. When modeling the bottom in Gemini, it is not possible to establish three dimensional bottom shapes that are in equilibrium. It is possible to fill an arbitrary shape in the Euler grid with a soil material. Two limitations prohibit the soil from staying in the arbitrary shape. The first is the density mismatch between the soil and water EOS. Since the 
Table 3

Dimensions of bottom contour shapes

\begin{tabular}{lcccccc}
\hline Figure & $\mathrm{X} 1(\mathrm{~m})$ & $\mathrm{X} 2(\mathrm{~m})$ & $\mathrm{Y}(\mathrm{m})$ & $\mathrm{Z} 1(\mathrm{~m})$ & $\mathrm{Z} 2(\mathrm{~m})$ & $\mathrm{Z} 3(\mathrm{~m})$ \\
\hline 8: Flat & 84.02 & N/A & 84.02 & 35.00 & 5.00 & N/A \\
9: Deep V & 42.01 & 42.01 & 84.02 & 25.00 & 20.00 & 5.00 \\
10: Inverted V & 42.01 & 42.01 & 84.02 & 25.00 & 20.00 & 5.00 \\
11: U Channel & 21.01 & 42.01 & 84.02 & 10.00 & 25.00 & 5.00 \\
12: Ramped & 84.02 & N/A & 84.02 & 25.00 & 20.00 & 5.00 \\
13: Anechoic & 84.00 & N/A & 84.00 & 30.00 & 10.00 & 5.00 \\
\hline
\end{tabular}

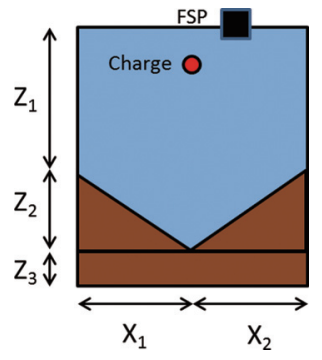

Fig. 9. Deep V.

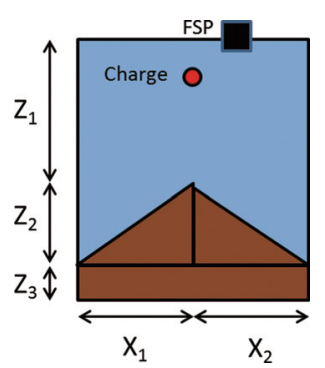

Fig. 10. Inverted V.

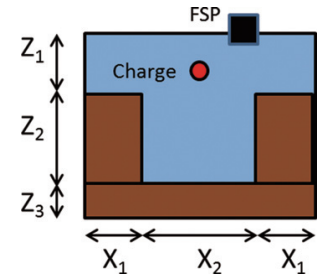

Fig. 11. U Channel.

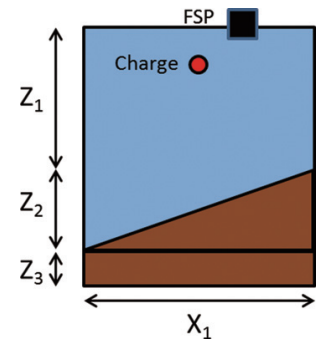

Fig. 12. Ramped.

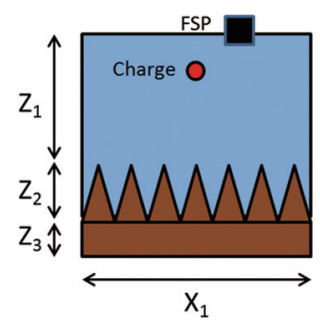

Fig. 13. Anechoic Pyramids.

soil is treated as an inviscid Eulerian fluid, over time the denser soil will settle to the bottom of the fluid geometry. The second limitation is the inability to equalize the pressure between the two dissimilar EOS layers. In contrast, modeling the bottom as a structural element in Dyna_N avoids the Gemini limitations entirely. The method for creating a stable, equalized bottom structure is the same whether it is flat or contoured.

In most deep water UNDEX events, the contour of the ocean bottom is trivial as the bottom reflection of the shockwave is of minimal magnitude. This assumption may not be true for littoral waters. The ability to model contoured shallow water environments could prove vital in determining the true nature of UNDEX effects on ships operating in these waters. With this in mind, five different bottom contours and one flat bottom model were developed and simulated with the FSP serving as the ship model. The goal was to determine the effect which bottom geometry had upon the response of both the fluid domain and the FSP. The bottom contours used are diagramed in Figs 8, 9 , 10, 11, 12 and 13. The associated dimensions are listed in Table 3.

Again, in all cases the standard charge of 60 pounds $(27.2 \mathrm{~kg}) \mathrm{HBX}-1$ at a depth of ten meters was placed with six meters of lateral separation from the FSP. The dimensions of the fluid domain were 84 meters in both the X and Y directions with the charge placed in the center. The depth of the fluid extended to one meter beyond the lowest point of the water-soil interface for each contoured model. Every simulation was run out to a full second to ensure that the response beyond the first bubble pulse was captured.

The finite element model of the FSP measured $487.68 \times 853.44 \mathrm{~cm}(16 \times 28 \mathrm{ft})$ with a weight of $5.07 \mathrm{e} 4 \mathrm{~kg}(111$, $821 \mathrm{lbs})$. Point masses were used to ballast to a depth of $121.92 \mathrm{~cm}(4 \mathrm{ft})$ for the draft. The structural materials consisted of varying thickness of steel from 0.4763 to $2.54 \mathrm{~cm}$ (3/16 to 1 in). In all there were 10,215 nodes and 11,172 shell elements with a nominal mesh spacing of $15.24 \times 15.24 \mathrm{~cm}(6 \times 6$ in $)$ in the FSP finite element model [14].

The initial analysis of the simulation focused on the vertical velocity of the FSP along with the corresponding fluid pressure directly beneath the FSP. Curiously, the velocity and pressure time history responses of every simulation were nearly identical as shown in Fig. 14. An examination of the pressure distribution through the water column for the flat bottom model at two different times in Figures 15 and 16 highlight the reason for the lack of differences.

Figure 15 displays the bottom reflection as it is returning to the surface. The white region just beneath the surface is the expected bulk cavitation zone. Eleven milliseconds later, in Fig. 16, the bottom reflection makes contact with the existing bulk cavitation zone. The result is rarefaction and the formation of a secondary bulk cavitation zone beneath the first. The initial FSP response is thus all but unaffected by the bottom reflection. 


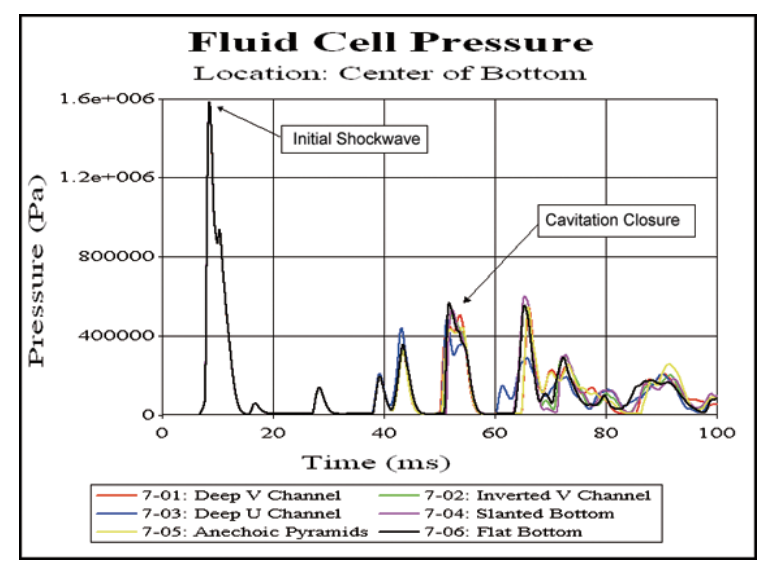

Fig. 14. Early pressure time history below the floating shock platform.

Table 4

Contoured bottom effect characterization at $5 \mathrm{~m}$ below FSP

\begin{tabular}{lccc}
\hline Contour & $\begin{array}{c}\text { B.R. Pressure } \\
\text { (\% of Max) }\end{array}$ & $\begin{array}{c}\text { B.R. Arrival } \\
\text { time (msec) }\end{array}$ & $\begin{array}{c}\text { Pulse } \\
\text { frequency }(\mathrm{Hz})\end{array}$ \\
\hline Flat & 12.6 & 37.5 & 1.77 \\
V Channel & 12.6 & 44.0 & 1.76 \\
Inverted V & 9.2 & 26.0 & 1.74 \\
U Channel & 13.6 & 37.5 & 1.75 \\
Ramped & 14.6 & 36.0 & 1.76 \\
Anechoic & 4.9 & 37.5 & 1.72 \\
\hline
\end{tabular}

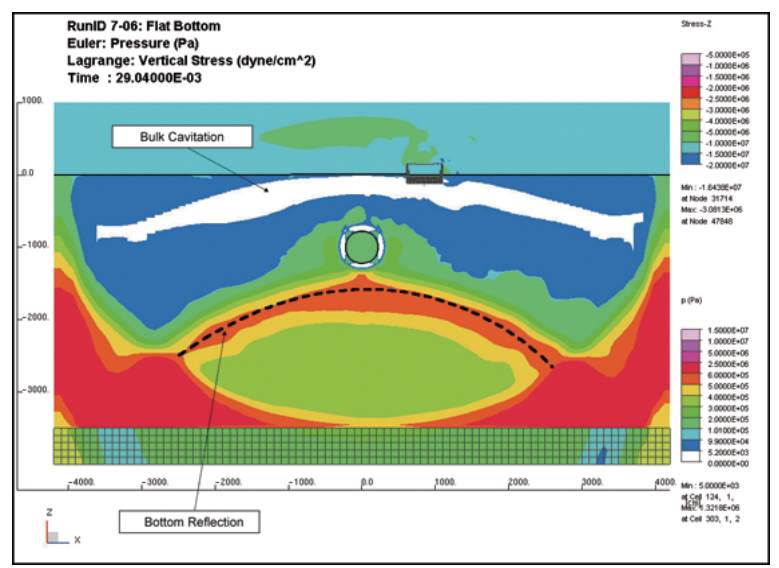

Fig. 15. Flat bottom pressure at $29 \mathrm{msec}$.

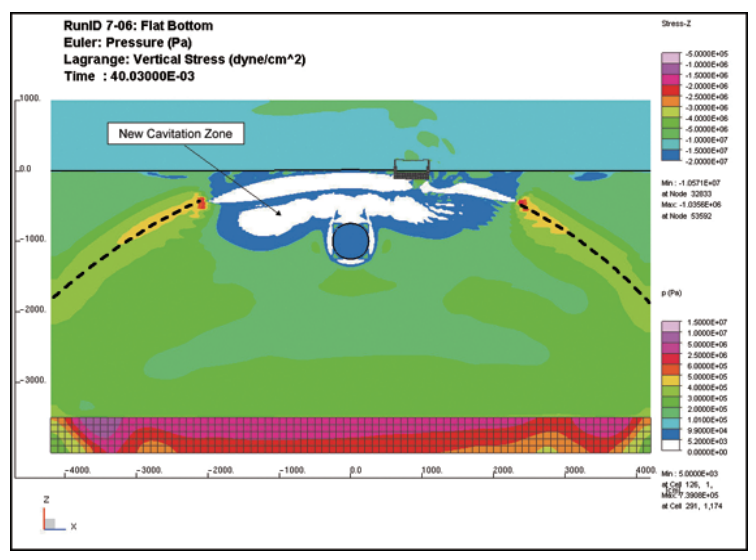

Fig. 16. Flat bottom pressure at $40 \mathrm{msec}$.

The Euler Clay and Reflective Boundary simulations of the same flat-bottom geometry displayed the creation of this second bulk cavitation zone as well. In each case the FSP response was insulated from the impact of the bottom reflection by the presence of the initial bulk cavitation zone. The results were compared with an open ocean simulation of the same geometry, charge, and FSP conducted by Didoszak, Prendergast, and Shin [13]. The FSP response in the first 100 milliseconds is nearly identical. This confirms that the initial bulk cavitation zone acts as a buffer. The creation of additional cavitation zones by the bottom reflection has been observed in both experimental and simulated data by Wardlaw and Ilamni [15]. This conclusion validated the analysis of the FSP response for the various bottom contours, which found negligible differences in the initial FSP response. This effect is strictly a by-product of this particular simulation geometry, upon which every simulation was based. The variation of bottom depth, charge weight, target position, and target draft would diminish the buffer effect of the bulk cavitation zone.

Using the existing simulations, the pressure histories of identical positions located at a depth of 5 meters below the FSP in each simulation was examined. The three data points examined in each simulation were the pressure and arrival time of the bottom reflection and the $1^{\text {st }}$ bubble pulse frequency. The arrival time is directly proportional to the distance the pressure wave had to travel. The summary of each simulation is listed in Table 4.

Using the Flat bottom contour as a control, Table 4 reveals the impact the various contours have on the bottom reflection. The V Channel focused the reflected wave such that even though the reflection traveled further up the water column by a distance of 10 meters, the pressure magnitude was equal to that of the Flat bottom case. Conversely, the Inverted $\mathrm{V}$ reflection traveled a shorter distance overall, but the bottom contour in this case spread the shockwave and decreased the pressure at the target point. In the Ramped contour the wave traveled a shorter distance as compared to the Flat bottom case without spreading and thus returned with a pressure greater than the Flat bottom. The 
Anechoic Pyramids bottom returned a pressure wave that was only $40 \%$ of the flat bottom reflection.

The fluid domain analysis additionally examined the differences in the first bubble pulse due to the bottom contour. While the magnitude appears unaffected, the timing of the pulse shows significant differences for the various contours. Once again, the two contours that showed the most significant difference from the flat bottom were the Anechoic Pyramids and the Inverted V. These differences in pulse timing can have significant impact on the total destructive effect of a charge on a target. The majority of UNDEX weapons are designed such that their bubble pulses are timed to excite the natural bending frequency of the ship, thereby causing resonance and increased damage [16].

While the bubble frequency variations in these 60 pound $(27.2 \mathrm{~kg})$ charge simulations were small, the charge weights of a majority of UNDEX threats are one to two orders of magnitude larger. This has the potential to magnify the bubble frequency variations due to bottom contours and could significantly alter the UNDEX weapon's effect on the target.

\section{Summary}

A method to model the ocean bottom as a Lagrangian solid was developed for comparison to the current Euler fluid bottom modeling approaches. Several sets of boundary and initial conditions were simulated to determine which combination introduced the least artificiality to the fluid domain solution. If an elastic or elasto-plastic model was used, it was then possible to apply non-reflecting boundary segments and nodal reaction forces at the boundaries along with Dyna_N Prestress. The non-reflecting boundary segments allowed the Lagrangian solid bottom to act as a semi-infinite domain, thereby eliminating retransmission waves. The Dyna_N Prestress imposed the hydrostatic loading and deformation on the bottom in order to minimize the magnitude of the initial bottom wave. This combination provided an accurate and efficient solution. However, the non-reflecting boundary segments and Dyna_N Prestress have limitations that prohibit their application to more complicated soil material models.

The validation of the Lagrangian solid bottom model was completed by comparing its fluid domain response to two existing bottom modeling methods. The first bottom modeling method was a purely reflective Eulerian boundary condition. The second method was the use of an Eulerian equation of state for a generic clay soil. The simulation geometry was consistent with the MIL-S-901D shock testing utilizing the Floating Shock Platform. Although the magnitude was greater, the bottom reflection of the Lagrangian solid bottom model had the same characteristic shape of the clay soil reflection. The first bubble pulse occurred 13 milliseconds sooner with the solid bottom than with the Euler clay soil. Overall, the fluid response of the simulation with a Lagrangian solid bottom was consistent with the Euler bottom modeling method.

One of the distinct advantages of using a Lagrangian solid bottom is the ability to model contoured bottom shapes. Six contoured solid bottom models were developed to investigate bottom contour effects on shallow water UNDEX events. The initial analysis of the FSP response showed only slight differences between the various contour models. This was caused by the buffer created by the bulk cavitation zone. The effect was specific only to the particular geometry selection. Modifications of the charge size, target separation, or bottom depth could diminish the effect, but to what extent is currently unknown. This is left for future study. Further investigation of the fluid domain response revealed that there were indeed significant differences between the initial bottom reflections for the different contours. The most important bottom contour effect was the distortion to the gas bubble and its associated first pulse timing. These changes could potentially have severe implications in the case of undersea weapons designed to take advantage of ship whipping.

\section{References}

[1] R.O. Work, Naval transformation and the littoral combat ship, Center for Strategic and Budgetary Assessments, 2004.

[2] U.S. Government, Accountability office. Improved littoral war-fighting capabilities needed: Report to congressional committees. (GAO Publication No. GAO-01-493), 2001.

[3] J.M. Didoszak, Y.S. Shin and D.H. Lewis, Shock trial simulation for naval ships, in: Proceedings of ASNE Day, 2004, IOS press, Amsterdam. 
[4] F.A. Costanzo, J.L. Baker, R.C. Javier, G.S. Harris, A. Goldberg and A. Meissner, Lütjens quick look report volume II: Test results and comparisons with pre-test DYSMAS simulations for shot C: Large charge shock test, Naval Surface Warfare Center Carderock, Carderock, NSWCCD-66-TR-2007/01, 2007.

[5] R. Ilamni, Analyses of the baltic sea test site UNDEX shock environment, Naval Surface Warfare Center Indian Head, Indian Head, Report IHTR 2785, 2006.

[6] R. McKeown, O. Dengel, G. Harris, H.-J. Diekhoff et al., Development and evaluation of DYSMAS hydrocode for predicting underwater explosion effects, volume I: Executive summary, Naval Surface Warfare Center, Indian Head, Report IHTR $2494,2004$.

[7] JASON Program Office, Navy ship underwater shock prediction and testing capability study, McLean, VA, the MITRE Corp., JSR-07-200, 2007.

[8] A.B. Wardlaw, J.A. Luton, J.R. Renzi, K.C. Kiddy and R.M. McKeown, The gemini euler solver for the coupled simulation of underwater explosions, NSWC Indian Head, Indian Head, Report IHTR 2500, 2003.

[9] A.B. Wardlaw, R. McKeown and H. Chen, Implementation and application of the p-alpha equation of state in the DYSMAS code, NSWC Dahlgren, Dahlgren, Report NSWCDD/TR-95/107, 1996.

[10] Naval surface warfare center indian head division, Gemini: The DYSMAS eulerian solver: User's Manual, Naval Surface Warfare Center, 2010.

[11] J.O. Hallquist, LS-DYNA theoretical manual, Livermore Software Technology Corporation, 1998.

[12] K. O'Shea and R. Koltuniuk, Structural dynamic analysis of parker dam with spillway, in: The Role of Dams in the 21st Century, San Antonio, 2006. IOS press, Amsterdam.

[13] J.M. Didoszak, G.R. Prendergast and Y.S. Shin, Parametric study in the m\&S of fully-coupled eulerian-lagrangian fluid structure interaction ship shock problems, The 79th Shock and Vibration Symposium Proceedings, Orlando, 2008.

[14] G.R. Prendergast and Y.W. Kwon, Optimizing the modeling and simulation of underwater explosion gas bubble dynamics in the DYSMAS hydrocode, The 81st Shock and Vibration Symposium Proceedings, Orlando, 2010.

[15] A.B. Wardlaw and R. Ilamni, Simulation of underwater explosion cavitation phenomena, NSWC Indian Head, Indian Head, Report IHTR 2589, 2004.

[16] A.H. Keil, The response of ships to underwater explosions, DTMB West Bethesda, Report 1576, 1961. 

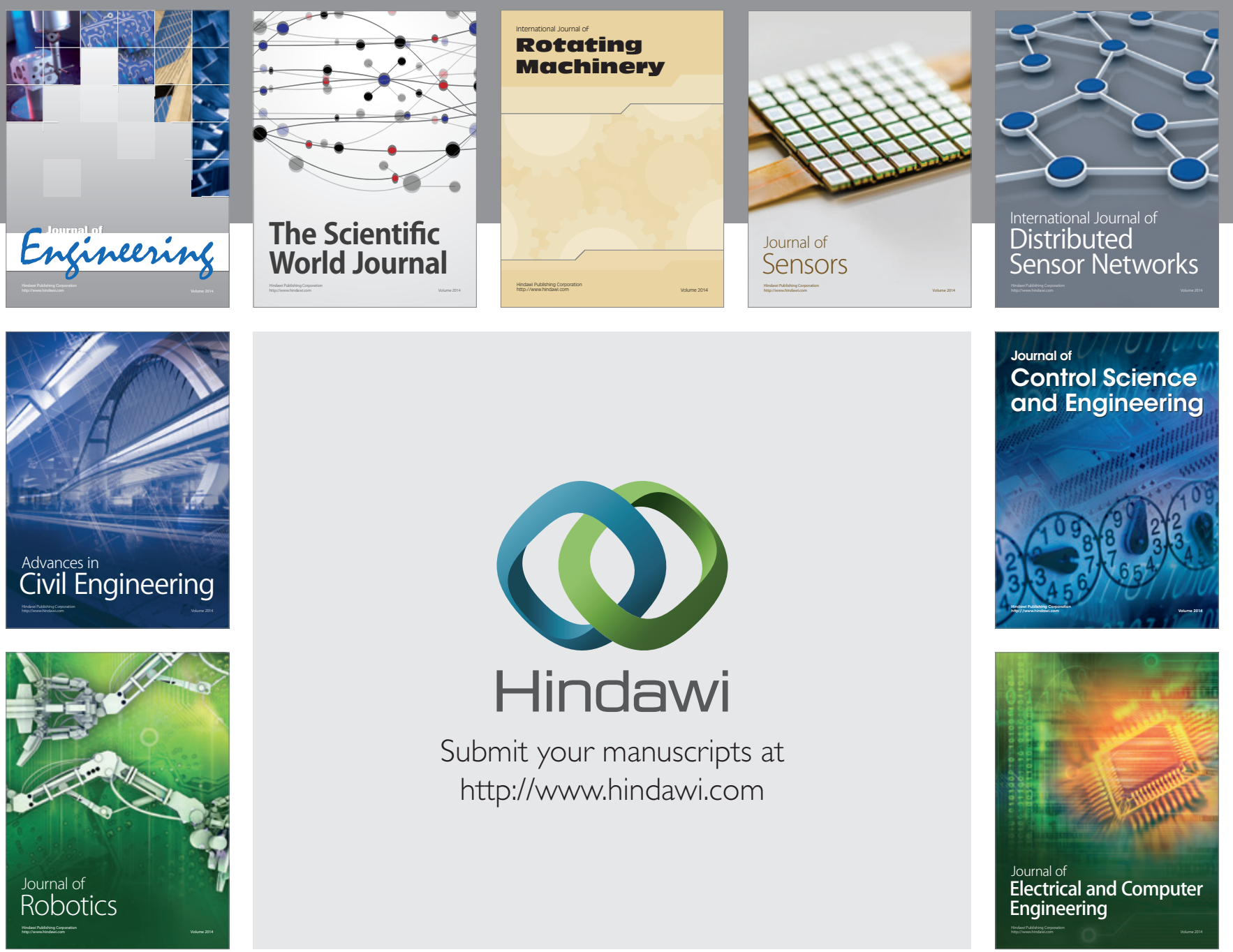

Submit your manuscripts at

http://www.hindawi.com
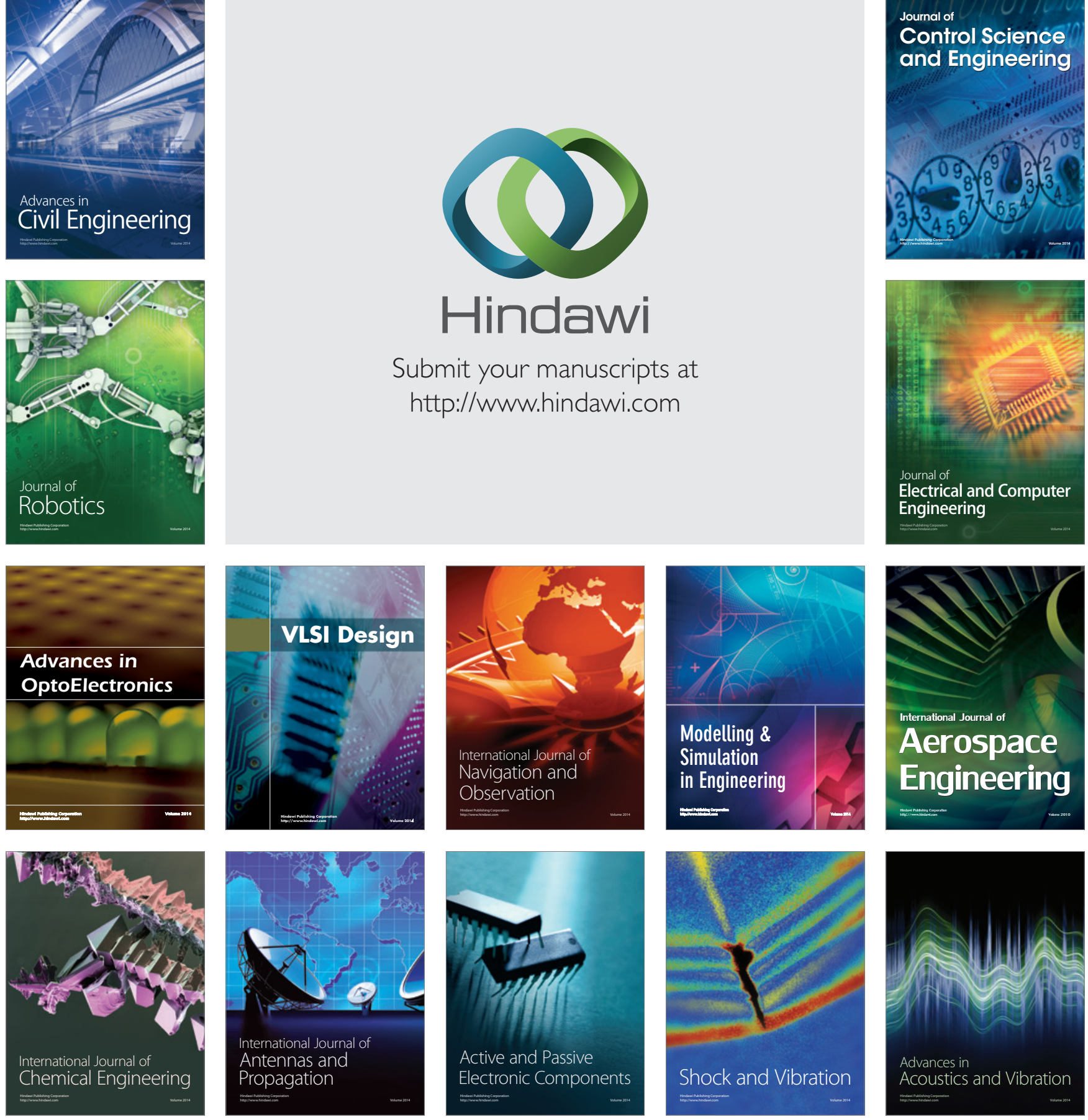